\title{
Detection of Mosquito Borne Disease in Human Blood using Image Processing Technique
}

\author{
Shruti Mardolkar \\ ETC Department \\ Goa College of Engineering Goa, India
}

\author{
H. G. Virani, PhD \\ ETC Department \\ Goa College of Engineering Goa, India
}

\begin{abstract}
Malaria is a life-threatening disease which is caused by the plasmodium parasites and is transmitted in human blood through the bite of a female ANOPHELES mosquito. It is a dreadful disease and may even lead to death if not rapidly diagnosed. This project aims an automated system which will detect malaria parasite in human blood. There are basically four types of malaria namely, P.falciparum, P.vivax, P.ovale and P.malariae. Image processing technique is used in this proposed system thus automating the detection process. This method involves steps like image acquisition, pre-processing, segmentation, feature extraction and classification. The features such as shape, size, standard deviation, skewness, kurtosis is extracted from the segmented image and are used in the classification stage in order to give more accurate results. The type and the stage of malaria parasite will be determined using multi-stage support vector machine. Most of the previous methods have been limited to detection of either one or two types of malaria listed above i.e. P.falciparum, P.ovale, P.vivax, P.malariae but this thesis aims in detecting the fifth type of malaria i.e. P.knowlesi which is now spreading rapidly all over the world thus taking the classification process one step further in the field of research.
\end{abstract}

\section{General Terms}

Image acquisition, pre-processing, segmentation, feature extraction, classification.

\section{Keywords}

Anopheles, support vector machine, P.falciparum, P.vivax, P.ovale, P.malariae, P.knowlesi.

\section{INTRODUCTION}

Malaria is a very dangerous disease which can lead to millions of deaths if not detected at an earlier stage. People with malaria often experience fever, chills and flu like illness. According to [2] in 2016 an estimated 216 million cases occurred worldwide and 4,45,000 people died, mostly children in African region. About 1700 cases of malaria are diagnosed in the United States each year. According to [3] malaria infected people in the year 2013 is $300-500$ million. The life cycle stages of malaria parasite are primarily described into three stages. The host female anopheles mosquito injects the sporozoites into the blood from their saliva. These sporozoites then enter into the liver and assault the liver cells. The invented liver cells then contain the fully grown sporozoites which later burst into merozoites and the merozoites are spread all over the blood infecting the red blood cells.

These merozoites give rise to the trophozoites, schizonts and gametocytes which are considered as the life cycle stages of malaria parasite.

There are basically four types of malaria namely, Plasmodium falciparum, Plasmodium vivax, Plasmodium ovale and
Plasmodium malariae. Among these 4 types of malaria P. falciparum is most common and most dangerous type of malaria.

In addition, Plasmodium knowlesi is another type of malaria which is spreading largely all over the world. Plasmodium knowlesi is a malaria parasite that is found in long-tailed and pig-tailed macaques. Naturally acquired human infections were thought to be extremely rare until a large focus of human infections was reported in 2004 in Sarawak, Malaysian Borneo. Human infections have now been described throughout Southeast Asia, and P.knowlesi is now recognized as the $5^{\text {th }}$ species of plasmodium causing malaria in human[1].

Also, there are three different stages to each of these five types of malaria i.e. trophozoites, schizonts and gametocytes;

Trophozoites stage- at this stage the parasite is in a ring shape with small one or two cytoplasm dots. At this stage the size of the RBC's infected by P.falciparum is $1-2$ um(micrometer), P.vivax is 2-3um, P.malariae is $1.33-1.5 u m$, P.ovale is $2.5 \mathrm{um}$ and P.knowlesi is 3-5um.[2][4]

Schizonts stage- these are matured trophozoites which can be recognized by the circular shape with many cytoplasm dots in it. At this stage the size of the RBC's infected by P.falciparum is 3-4um, P.vivax is 9um, P.malariae is 2.5-4um, P.ovale is 34um and P.knowlesi is 5-6um.[2][4]

Gametocytes stage- this stage is known as the sexual growth stage and the male female parasites are formed in this stage. The male size is smaller than the female gametocytes which are somewhat banana shaped. At this stage the average size of the RBC's infected by P.falciparum is 7-14um, P.vivax is 1016um, P.malariae is 4.5-6.75um, P.ovale is 9-13.5um and P.knowlesi is 7-8um [2][4].

Figure 1 shows the different life cycle stages of P.falciparum, P.vivax, P.malariae, and P.ovale [14] and Figure 2 shows the life cycle stages of P.knowlesi [15].

\section{LITERATURE REVIEW}

There are different methods proposed by different researchers to detect malaria in blood smears using image processing technique. Some of them are; S.S. Savkare used otsu thresholding, watershed transform and SVM classifier to detect red blood cells with consecutive classification into parasite infected and normal cells for estimation of parasitemia[5].

F.B Trek and A.G Dempster used color normalization to maintain illumination and color constancy and also used Bayesian decision rule to classify stained/non-stained pixels which gave satisfactory results [6].

Subhamoy Mandal presented an optimized normalized cut method for segmentation and also used different color space like RGB, HSV, YCbCr and NTSC and concluded that HSV 
is the best channel for detection, the efficiency was tested using Rand's index [7].

Miss kshipra Charpe used Annular Ring Ratio (ARR) transform so as to avoid the miscount of parasites in blood samples due to the overlapping of cells. Also, many features such as shape, size, intensity etc were considered to get highly accurate results [8].

Akshay Randive used an algorithm which shows areas where it believes to be infected by malaria in black. Using this algorithm, it was detected that the sample of patient is Plasmodium vivax which consist of an elongated and large cell size with a dot and ring inside the cell [9].

Pallavi Suradkar proposed an algorithm which detected malaria by counting malaria parasites in blood smear images, the steps followed in the algorithm were Image segmentation smoothing processing and gradient edge detection. It was identified that there exists a ring-shaped parasite on RBC's and the ring is generally blue in color [10].

Hanung Adi Nugroho proposed image enhancement, k-means clustering and histogram-based features and identified that the blood smear is infected by Plasmodium falciparum [11].

Silvia et. al proposed a technique for estimating parasitemia. Estimation is carried out by using template matching for detection of RBC's, parasites are detected using variancebased technique for grayscale images and second approach is based on color co-occurrence matrix. SVM classifier is used for classification purpose [12].

K.M. Khatri used color normalization, morphological operations, RBC counting and contour formation for counting the parasites in blood smear images and achieved good results

P. falciparum P. vivax P.malariae P. ovale

Rings
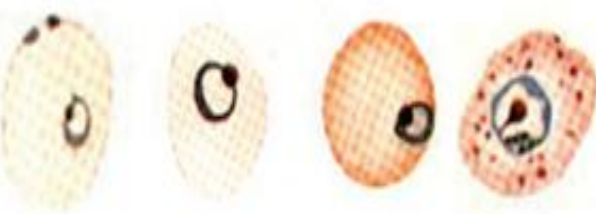

Schizonts
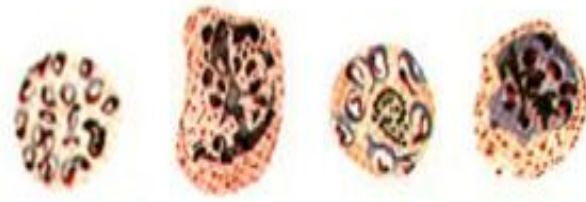

Gametocytes
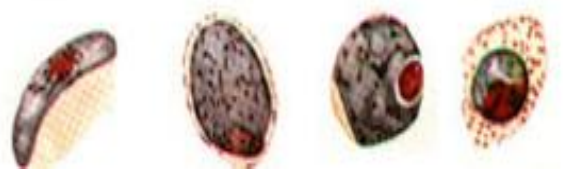

Figure 1: life cycle stages of different types of malaria [14]

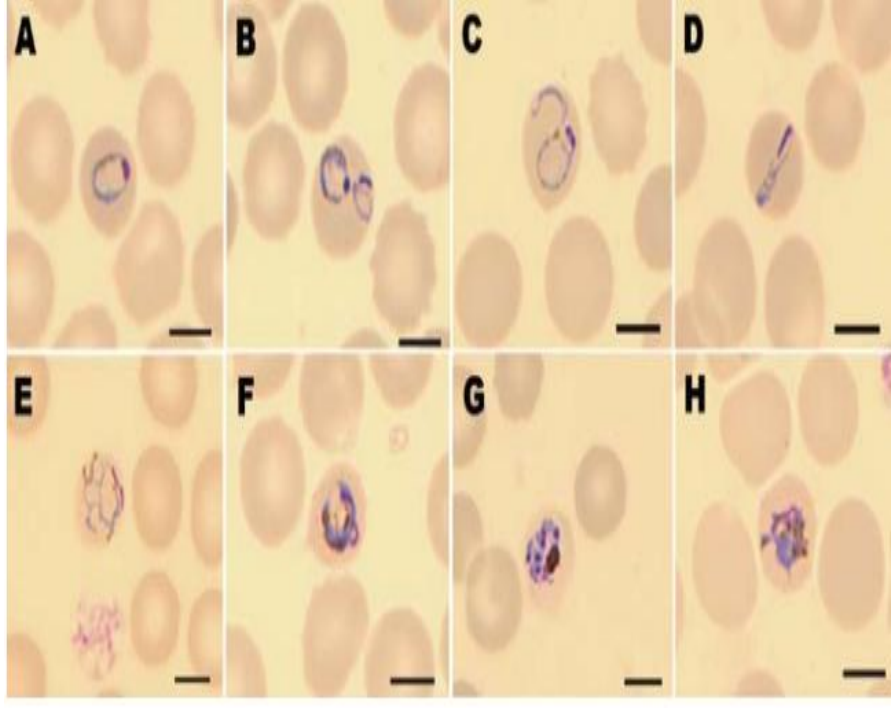

Figure 2: life cycle stages of P.knowlesi, image shows the examples of trophozoites (A-F), $\operatorname{schizont}(G)$, gametocyte(H) [15].

\section{CLINICAL METHODS}

Clinically malaria in human blood is detected by counting and analyzing the parasites manually. Manual microscopy is carried out by examining thin blood films on slides under the microscope and reporting the percentage of parasitemia (i.e. number of infected red blood cells for over 100 microscopic fields). Giemsa staining is most widely used to highlight the parasites. The disadvantage of giemsa is that it also stains other blood film particles such as white blood cells, platelets, and side artefacts (dust particles). Thus, manual detection of malaria requires extensive human intervention during the diagnostic process which can often lead to late and sometimes erroneous diagnosis [1].

\section{METHODOLOGY}

Figure 3 shows the detailed steps that have been followed in this thesis to detect and to classify the type and stage of malaria.

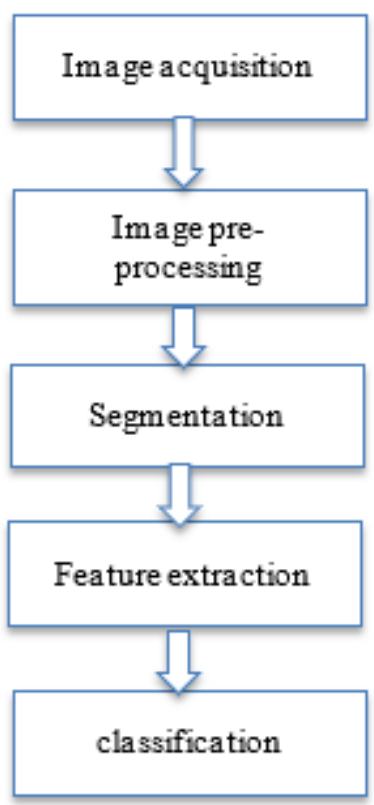

Figure 3: flow process 


\subsection{Image acquisition}

Images of human blood smears infected by malaria parasites (P.falciparum, P.vivax, P.ovale, P.malariae, P.knowlesi) are obtained from online data base[2] [4].

\subsection{Image pre-processing}

Main aim of image pre-processing is to improve the image data that is, to suppress unwanted distortions or enhance some image features important for further processing.

In this step the colored image is converted into the greyscale image in order to analyze the intensity which is further required to calculate the different features of the blood smear image.

\subsection{Image segmentation}

Segmentation is often considered to be the first step in image analysis technique. The purpose of segmentation is to subdivide an image into meaningful non-overlapping regions, which would be used for further analysis (i.e. feature extraction and classification of life cycle stages of malaria parasite). As malaria parasites are injected in human blood, they infect the RBC's (red blood cells) and multiply in RBC's only. Therefore, the RBC's has to be segmented from other artefacts (WBC, platelets, dirt, dye crystals etc).

Also, the RBC's are located very close to each other, or sometimes are overlapping each other, which leads to difficulty in analysing the RBC's. Hence in this step of image processing the separation of RBC's and the artefacts is performed. In this study the steps performed under segmentation are as follows:

Step 1- Canny edge detection method is used to detect wide range of edges of the objects present in the blood smear image. Though canny edge detection takes some time to execute, it is still preferred over other methods (e.g. Sobel edge detection) because it is very accurate even in the presence of noise.

Step 2- After edge detection, Dilation is carried out for filling the holes in the image.

Step 3- Final step in segmentation is Erosion in which the size of unwanted objects is reduced.

\subsection{Feature extraction}

Features are the main distinguishing points of any object. To distinguish the infected RBC from the non-infected RBC, we have to consider some features which will help us to detect the malaria and its different stages. [8] Following are the features on which this work is being focused on:

\subsubsection{Size}

The cell size is the total number of pixels of the area of the infected cells.

\subsubsection{Shape}

The shape of the parasite will change accordingly at different stages of life cycle of the parasite. In this project shapes are detected using Hough transform which gives more effective results. Hough transform performs this function by grouping the edge points into objects.

\subsubsection{Texture features}

The texture features are calculated using GLCM (Gray-Level Co-occurrence Matrix). Given an image composed of pixels each with an intensity (a specific gray level), the GLCM is a tabulation of how often different combinations of gray levels co-occur in an image. GLCM basically calculates the intensity of a pixel and its neighbouring pixel. Different texture-based features which are calculated using GLCM are: contrast, entropy and energy which is given as

\subsubsection{Contrast}

It is a measure of the amount of local variations present in an image.

\subsubsection{Entropy}

It is used to measure the randomness or disorder of an image.

\subsubsection{Energy}

It is used to measure the uniformity of an image.

\subsection{4 mean}

It gives an estimate of average intensity level in the region of the cell.

\subsection{5 skewness}

It describes how symmetric the intensity distribution is about the curve. Skewness of a normal red blood cell is up to 2 and for infected cell it is above 2 .

\subsection{6 kurtosis}

It describes how flat the intensity distribution is about the mean. Kurtosis of a normal red blood cell is up to 3 and of infected cell it is above 3 .

\subsection{7 standard deviation}

It gives how much the distribution differs from the mean. Standard deviation of an infected cell is above 2 .

\subsection{Classification}

The SVM classifier is used to classify the type and the stage of the malaria. It consists of the training set and the test set. When a test image is selected the classifier compares the features of the training set with the test set and classifies the type and the stage of malaria. The advantage of SVM classifier over other classifiers is that there are no upper limits on the number of features used also, it is highly accurate and fast.

\section{RESULTS}

The data set consists of 300 images of blood smears which are infected by different types and different stages of malaria. The segmentation outputs obtained are shown in figures 4,5,6,7,8.

Table 1 shows the features extracted from various stages of P.falciparum similarly tables 2,3,4,5 shows the features extracted from various stages of P.vivax, P.malariae, P.ovale and P.knowlesi respectively. 


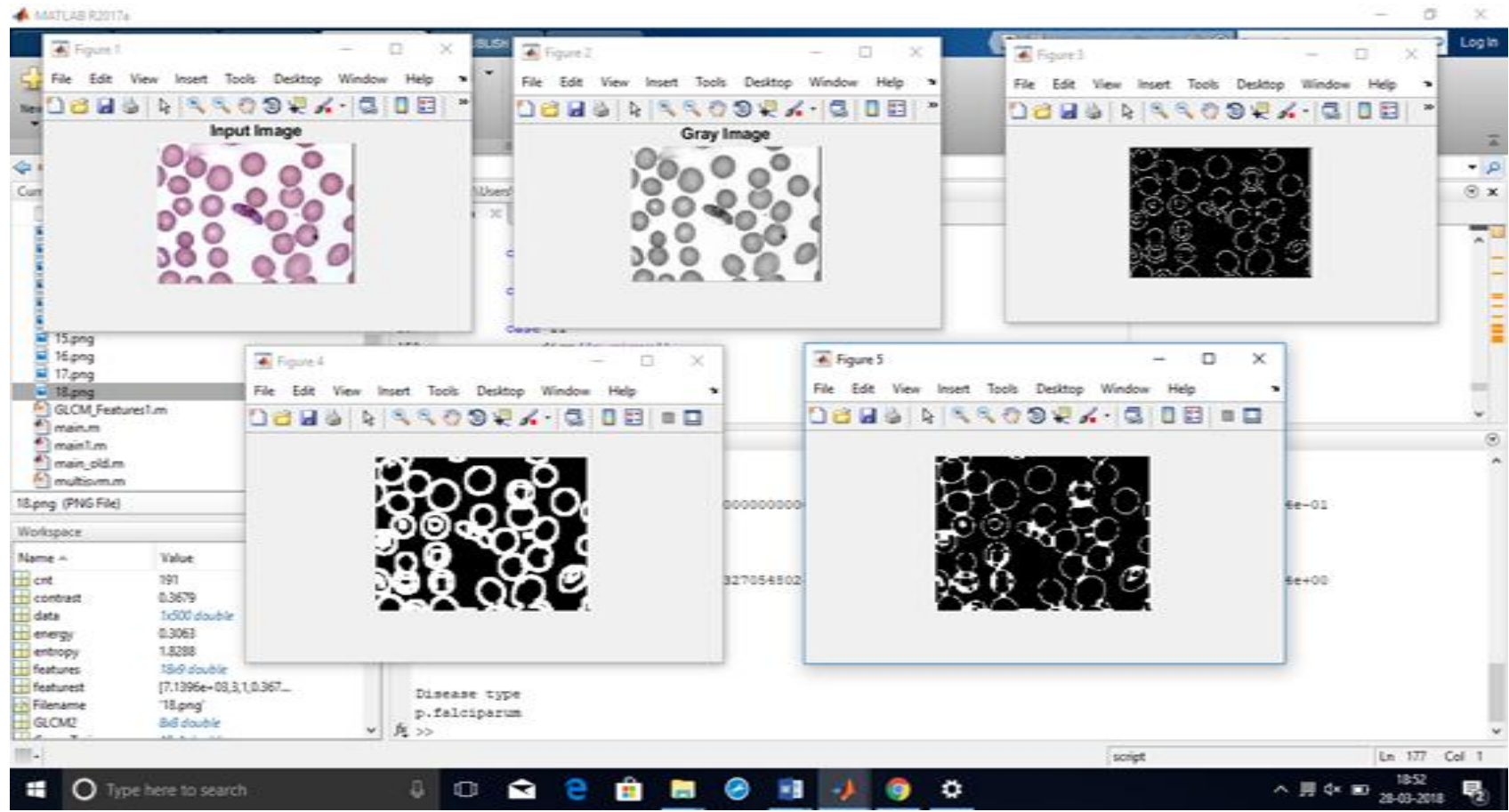

Figure 4: segmentation of blood smear images and classification of malaria using SVM classifier(output shows P.falciparum)

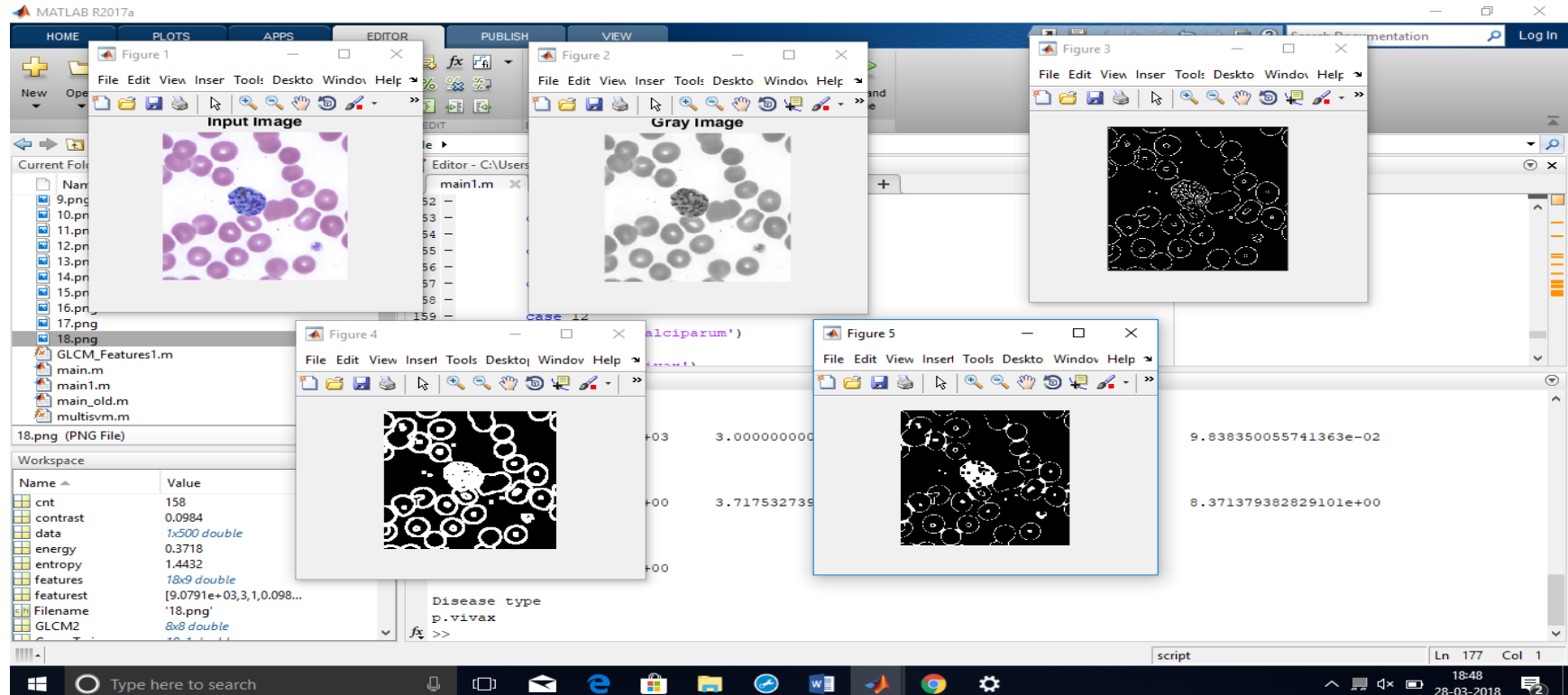

Figure 5: segmentation of blood smear images and classification of malaria using SVM classifier(output shows P.vivax) 


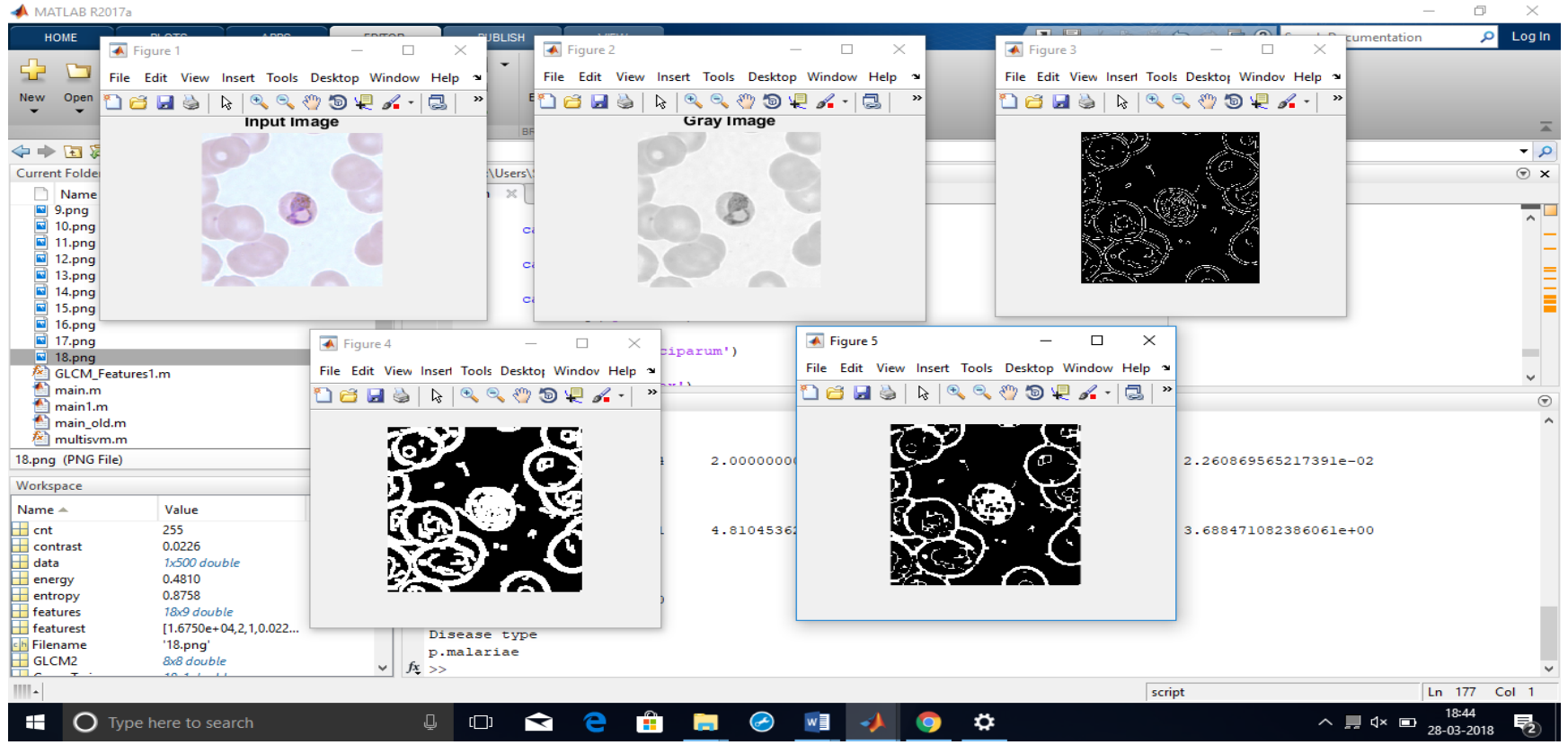

Figure 6: segmentation of blood smear images and classification of malaria using SVM classifier(output shows P.malariae)

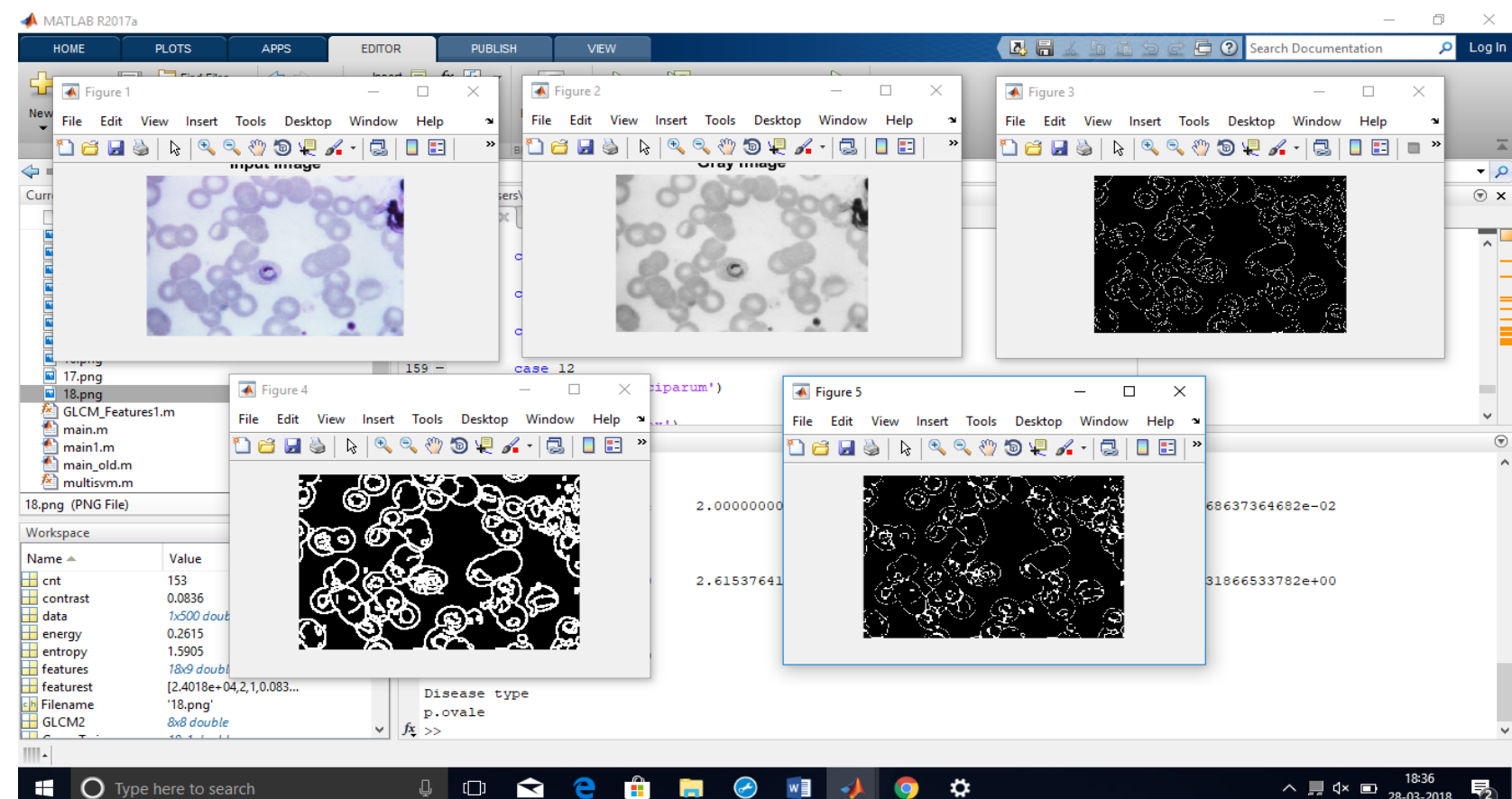

Figure 7: segmentation of blood smear images and classification of malaria using SVM classifier(output shows P.ovale) 


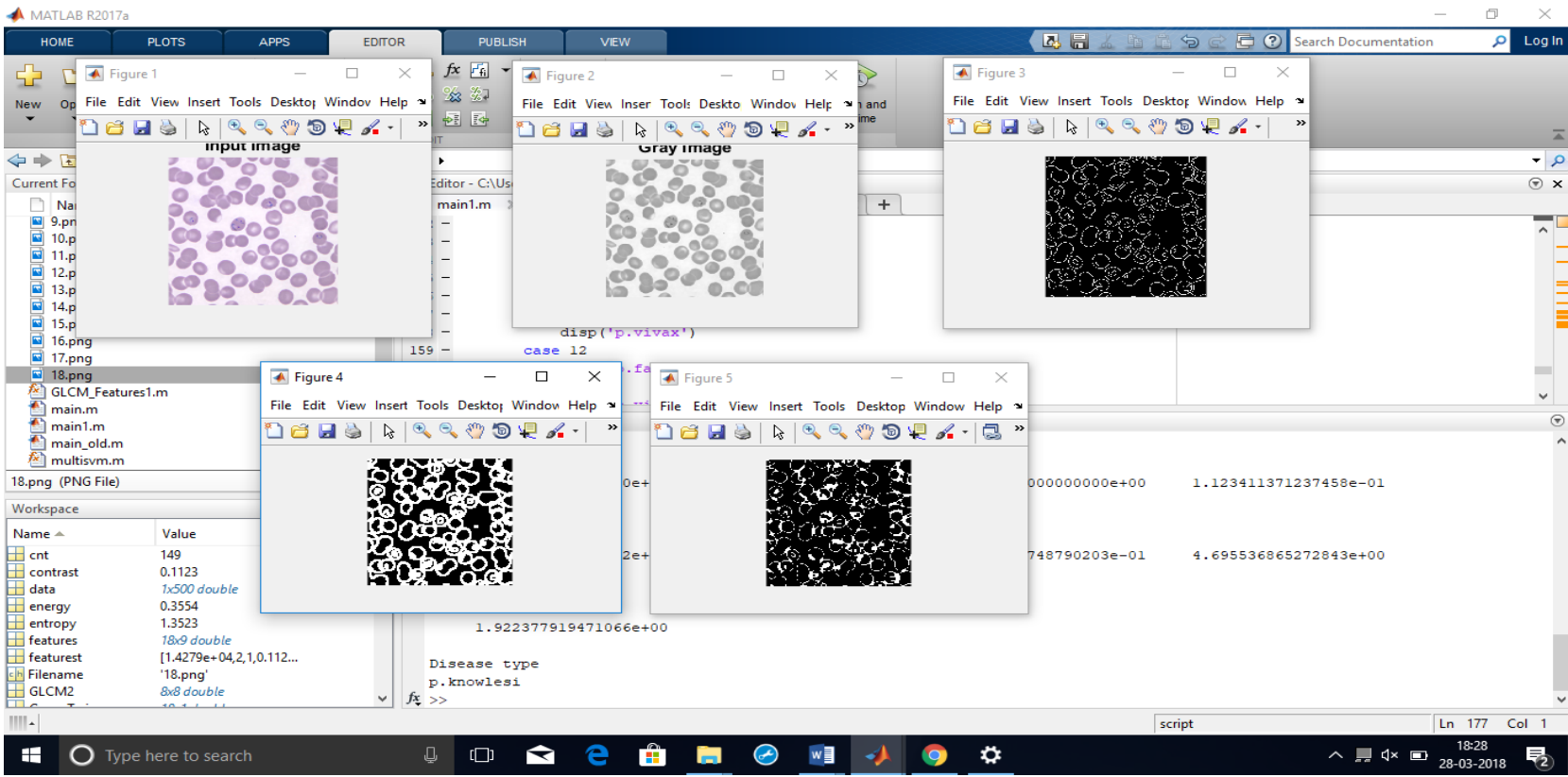

Figure 8: segmentation of blood smear images and classification of malaria using SVM classifier(output shows P.knowlesi)

Table 1: features extracted from P.falciparum and its different stages(trophozoite, schizonts, gametocytes)

\begin{tabular}{|l|l|l|l|}
\hline $\begin{array}{l}\text { Feature } \\
\text { extracted }\end{array}$ & $\begin{array}{l}\text { Trophozoite } \\
\text { Stage }\end{array}$ & $\begin{array}{l}\text { Schizont } \\
\text { Stage }\end{array}$ & $\begin{array}{l}\text { Gametocyte } \\
\text { Stage }\end{array}$ \\
\hline Size(micrometer) & 1.58 & 6.65 & 7.13 \\
\hline Shape & Round & Round & Oval \\
\hline Kurtosis & 9.00 & 4.68 & 5.43 \\
\hline $\begin{array}{l}\text { Standard } \\
\text { deviation }\end{array}$ & 2.88 & 3.60 & 3.44 \\
\hline Intensity & 1 & 1 & 1 \\
\hline Skewness & 2.82 & 2.2 & 2.10 \\
& & & \\
\hline
\end{tabular}

Table 2: features extracted from P.vivax and its different stages(trophozoite, schizonts, gametocytes)

\begin{tabular}{|l|l|l|l|}
\hline $\begin{array}{l}\text { Feature } \\
\text { extracted }\end{array}$ & $\begin{array}{l}\text { Trophozoite } \\
\text { Stage }\end{array}$ & $\begin{array}{l}\text { Schizont } \\
\text { Stage }\end{array}$ & $\begin{array}{l}\text { Gametocyte } \\
\text { Stage }\end{array}$ \\
\hline Size(micrometer) & 2.03 & 9.07 & 9.92 \\
\hline Shape & Round & Round & Round \\
\hline Kurtosis & 5.03 & 8.37 & 7.44 \\
\hline $\begin{array}{l}\text { Standard } \\
\text { deviation }\end{array}$ & 3.52 & 2.965 & 3.09 \\
\hline Intensity & 1 & 1 & 1 \\
\hline Skewness & 2.01 & 2.71 & 2.53 \\
\hline
\end{tabular}

Table 3: features extracted from P.malariae and its different stages(trophozoite, schizonts, gametocytes)

\begin{tabular}{|l|l|l|l|}
\hline $\begin{array}{l}\text { Feature } \\
\text { extracted }\end{array}$ & $\begin{array}{l}\text { Trophozoite } \\
\text { Stage }\end{array}$ & $\begin{array}{l}\text { Schizont } \\
\text { Stage }\end{array}$ & $\begin{array}{l}\text { Gametocyte } \\
\text { Stage }\end{array}$ \\
\hline Size(micrometer) & 1.67 & 2.55 & 6.27 \\
\hline Shape & Round & Oval & Oval \\
\hline Kurtosis & 3.68 & 8.16 & 5.87 \\
\hline $\begin{array}{l}\text { Standard } \\
\text { deviation }\end{array}$ & 3.86 & 2.99 & 3.357 \\
\hline Intensity & 1 & 1 & 1 \\
\hline Skewness & 2.10 & 2.67 & 2.20 \\
\hline
\end{tabular}

Table 4: features extracted from P.ovale and its different stages(trophozoite, schizonts, gametocytes)

\begin{tabular}{|l|l|l|l|}
\hline $\begin{array}{l}\text { Feature } \\
\text { extracted }\end{array}$ & $\begin{array}{l}\text { Trophozoite } \\
\text { Stage }\end{array}$ & $\begin{array}{l}\text { Schizont } \\
\text { Stage }\end{array}$ & $\begin{array}{l}\text { Gametocyte } \\
\text { Stage }\end{array}$ \\
\hline Size(micrometer) & 2.40 & 4.31 & 9.5 \\
\hline Shape & Round & Oval & Oval \\
\hline Kurtosis & 9.26 & 3.09 & 6.61 \\
\hline $\begin{array}{l}\text { Standard } \\
\text { deviation }\end{array}$ & 2.85 & 2.73 & 3.22 \\
\hline
\end{tabular}




\begin{tabular}{|l|l|l|l|}
\hline Intensity & 1 & 1 & 1 \\
\hline Skewness & 2.87 & 3.06 & 2.37 \\
& & & \\
\hline
\end{tabular}

Table 5: features extracted from P.knowlesi and its different stages(trophozoite, schizonts, gametocytes)

\begin{tabular}{|l|l|l|l|}
\hline $\begin{array}{l}\text { Feature } \\
\text { extracted }\end{array}$ & $\begin{array}{l}\text { Trophozoite } \\
\text { Stage }\end{array}$ & $\begin{array}{l}\text { Schizont } \\
\text { Stage }\end{array}$ & $\begin{array}{l}\text { Gametocyte } \\
\text { Stage }\end{array}$ \\
\hline Size(micrometer) & 4.01 & 5.42 & 7.4 \\
\hline Shape & Round & Round & Oval \\
\hline Kurtosis & 5.5 & 6.66 & 4.94 \\
\hline $\begin{array}{l}\text { Standard } \\
\text { deviation }\end{array}$ & 2.5 & 4.11 & 2.9 \\
\hline Intensity & 1 & 1 & 1 \\
\hline Skewness & 2.90 & 3.3 & 3.10 \\
\hline
\end{tabular}

\section{CONCLUSION AND FUTURE WORK}

This work mainly focuses on detection of malaria and its types, and as well as its stages. The results obtained are highly accurate since maximum number of features such as size, shape, texture and statistical features are extracted from the blood smear images and then the classification is carried out using the methodology as explained above. This algorithm will be classifying the types such as P.falciparum, P.vivax, P.malariae, P.ovale and the $5^{\text {th }}$ type of malaria that is P.knowlesi and also the different stages of each of these 5 types such as trophozoites, schizonts and gametocyte stage. This system further can be used for diagnosis of other hematological problems by making changes to the algorithm accordingly. Future work can be done to make the system online to diagnose malaria. Also, more segmentation algorithms can be explored so as to obtain more better results.

\section{REFERENCES}

[1] National Centre for Biotechnology Information

[2] Centers for disease control and prevention
[3] World Health Organization

[4] Public health image library

[5] S.S.Savkare,S.P.Narote, Automatic detection of malaria parasites for estimating parasitemia, 2011-International journal of computer science and security.

[6] F.B Trek, A.G Dempster, I.Kale, Malaria parasite detection in peripheral blood images, September 2006Proc.british vision conference.

[7] Subhamoy Mandal, Amit Kumar, J Chatterjee, M Manjunatha, Ajoy K Roy, Segmentation of blood smear images using normalized cuts for detection of malaria parsites, 2010 Indian conference, Annual IEEE.

[8] Miss Kshipra Charpe, Dr V.K Bairagi, Automated malaria parasite and their Stage detection in microscopic blood images, 2014- IEEE sponsored $9^{\text {th }}$ International conference on intelligent system and control.

[9] Akshay Randive, Amit Salunke, Detection of malaria parasite using image processing, April 2014International journal of advance engineering and research development volume 4 , issue 4 .

[10] Pallavi T. Suradkar, Detection of malaria parasite in blood using image processing, April 2013-International journal of engineering and innovative technology.

[11] Hancing Adi Nugroho, Son Ali Akbar, E.Elsa Herdiana Murhandarwati, Feature extraction and classification for detection malaria parasites in thin blood smear, International conference on information technology, computer and electrical engineering 2015.

[12] S. Halim et al., "Estimating malaria parasitemia from blood smear images", IEEE international conference on control, automation, robotics and vision, pp 1-6, 2006.

[13] K. M. Khatri, V. R. Ratnaparkhe, S.S. Agarwal, A.S. Balchandra, Image processing approach for malaria parasite identification, International journal of computer applications GOTETC-IP'13.

[14] www.malariasite.com

[15] Jaap J. van Hellemond, Marijke Rutten, Rob Koelewijn, Anne-Marie Zeeman, Jaco J. Verweij, Pieter J. Wismans, Clemens H. Kocken, and Perry J.J. van Genderen, Human plasmodium knowlesi infection detected by rapid diagnostic test for malaria. Emerging Infectious Diseases - www.cdc.gov/eid • Vol. 15, No. 9, September 2009 